\title{
Enhanced linkage of a locus on chromosome 2 to premature coronary artery disease in the absence of hypercholesterolemia
}

Jérémie Nsengimana ${ }^{1}$, Nilesh J Samani ${ }^{2}$, Alistair S Hall ${ }^{3}$, Anthony J Balmforth ${ }^{3,4}$, Massimo Mangino $^{2}$, Nadira Yuldasheva ${ }^{3}$, Azhar Maqbool ${ }^{3}$, Peter Braund ${ }^{2}$, Paul Burton ${ }^{5}$, D Timothy Bishop ${ }^{1}$, Stephen G Ball ${ }^{3}$ and Jennifer H Barrett ${ }^{*, 1}$, for the British Heart Foundation Family Heart Study Research Group

\footnotetext{
${ }^{1}$ Genetic Epidemiology Division, Leeds Institute of Molecular Medicine, University of Leeds, Leeds, UK; ${ }^{2}$ Department of Cardiovascular Sciences, University of Leicester, Leicester, UK; ${ }^{3}$ Institute for Cardiovascular Research, University of Leeds, Leeds, UK; ${ }^{4}$ Academic Unit of Molecular Vascular Medicine, University of Leeds, Leeds, UK; ${ }^{5}$ Department of Health Sciences, University of Leicester, Leicester, UK
}

Linkage studies of complex diseases have so far had limited success in producing significant and replicable results, in part owing to genetic heterogeneity. We recently reported the results of a large genome-wide linkage scan for coronary artery disease (CAD) based on 1933 families. The greatest evidence for linkage was to a region of chromosome 2, with a logarithm of odds (LOD) score of 1.86, based on the nonparametric $S_{A L L}$ statistic, which did not reach genome-wide significance $(P>0.3)$. Inclusion of a covariate in linkage analysis can be a powerful method of accounting for disease heterogeneity. As CAD is a heterogeneous disease, we carried out a linkage analysis of chromosome 2 incorporating covariates. Increased evidence for linkage was found when hypercholesterolemia was considered (LOD score including covariate of 4.4 ) reaching genome-wide significance as assessed by simulation $(P=0.04)$. Results showed that the original evidence for linkage was largely attributable to the subset of 108 nonhypercholesterolemic affected sibling pairs. In separate linkage analyses of subsets of hypercholesterolemic and non-hypercholesterolemic sibling pairs, the maximum LOD scores were 1.09 in the former group and 3.74 in the latter. This result illustrates the potential to increase the power of linkage analysis in the presence of heterogeneity by inclusion of covariates. This linked locus on chromosome 2 should now be investigated further to identify the gene(s) influencing risk of CAD in subjects with a normal level of total cholesterol. Candidate genes include the interleukin 1 cluster and two potential regulators of high-density lipoprotein cholesterol level, PLA2R1 and OSBPL6.

European Journal of Human Genetics (2007) 15, 313-319. doi:10.1038/sj.ejhg.5201752; published online 6 December 2006

Keywords: coronary artery disease; linkage; covariates; hypercholesterolemia

\footnotetext{
${ }^{*}$ Correspondence: Dr JH Barrett, Genetic Epidemiology Division, Leeds Institute of Molecular Medicine, St James's University Hospital, Beckett Street, Leeds LS9 7TF, UK. Tel: + 44113 3438637; Fax: + 44113 2340183; E-mail: j.h.barrett@leeds.ac.uk

Received 23 August 2006; revised 20 October 2006; accepted 25 October 2006; published online 6 December 2006
}

Introduction

We recently reported the results of a genome-wide linkage scan for premature atherosclerotic coronary artery disease (CAD) based on 4175 affected subjects from 1933 families. ${ }^{1}$ Despite the large size of the study, the strongest result was suggestive evidence for linkage on chromosome 2 , with a 
multipoint non-parametric logarithm of odds (LOD) score of 1.98 in an initial scan (pointwise $P=0.0014$, genomewide $P=0.31$, based on observing at least one LOD score of 1.98 or higher in 310 out of 1000 simulations) falling to 1.86 with the addition of extra markers in the region. Failure to find evidence of significant linkage at the genome-wide level is common in the analysis of complex diseases. This may be because the disease is influenced by interactions between distinct genetic loci, genetic heterogeneity or both. ${ }^{2,3}$ Genetic heterogeneity may explain why, among the analyses showing suggestive or significant linkage, a relatively small proportion has so far been replicated, even when estimates of sib recurrence risk ratio are high. ${ }^{4-6}$

One way of resolving the problem of heterogeneity is to use a more restrictive definition of phenotype at recruitment or to separately analyse subsets of the study participants with more homogeneous disease characteristics. For example, Wang et al. ${ }^{7}$ reported significant evidence of linkage to a locus on chromosome 1 by considering subjects with myocardial infarction (MI), diagnosed by age 45 years in males or 50 years in females. Applying narrow criteria at recruitment involves strong assumptions about which phenotypic groups are likely to be genetically homogeneous. On the other hand, separate analysis of subsets of study participants may lack statistical power to detect linkage. An alternative approach is to include suspected sources of heterogeneity as covariates in the statistical model. The use of covariates in linkage analysis has contributed to mapping or confirming the position of genes for prostate cancer, ${ }^{8,9}$ late-onset Alzheimer disease ${ }^{10,11}$ and recurrent early-onset depression. ${ }^{12}$ In this study, we report the results of a sibling pair linkage analysis of CAD on chromosome 2 using the British Heart Foundation (BHF) Family Heart Study including hypercholesterolemia as a covariate.

\section{Materials and methods Study subjects}

Data collection has been described in detail elsewhere. ${ }^{1}$ Briefly, the BHF Family Heart Study comprises 1933 families including 4175 persons affected with CAD (1675 affected sibling pairs, 220 affected trios and 38 sibships or extended families with more than three affected individuals). Affection with CAD was defined as having had one or more of these conditions before age 66 years: MI, angina, percutaneous transluminal coronary angioplasty or coronary artery bypass surgery. Clinical information available for all or the majority of patients include body mass index (BMI), age of CAD onset, and self-reported histories of smoking, hypercholesterolemia, diabetes and hypertension. Four hundred and sixteen microsatellites were genotyped $^{1}$ and the present covariate analysis is based on 38 of them, which cover chromosome 2 . Study participants reported clinical information about themselves by completing a questionnaire. The study was approved by the Yorkshire Multicentre Research Ethics Committee and by 206 local research ethics committees across the UK. For hypercholesterolemia, participants were asked whether their general practitioner (family doctor) had ever told them that they had a high cholesterol level and whether they had ever taken any lipid-lowering treatment.

\section{Statistical analysis}

To investigate the effect of heterogeneity on linkage of CAD to chromosome 2, regression analyses were carried out expressing allele-sharing probabilities as a function of a series of covariates, using the method proposed by Rice ${ }^{13}$ and extended by Holmans. ${ }^{12,14}$ The probability $P$ that a pair of siblings share identical-by-descent (IBD) an allele inherited from a particular parent at a putative disease locus is expressed as a function of the covariate $x$

$$
P=\frac{\exp (\alpha+\beta x)}{1+\exp (\alpha+\beta x)}
$$

The test statistic for linkage allowing for the covariate effect is based on a ratio of likelihoods:

$$
\mathrm{LOD}=\log _{10}\left[\frac{L(\hat{\alpha}, \hat{\beta})}{L(\alpha=\beta=0)}\right]
$$

Binary variables such as hypercholesterolemia give rise to a three-level factor $\beta$ for each sibling pair $(0,1$ or 2 hypercholesterolemics in the pair). Multipoint posterior IBD probabilities (based on the marker genotypes) were computed using the program Allegro. ${ }^{15}$ Regression analysis was carried out (computation of the LOD score of Eq. 2) with version 9 of STATA ${ }^{16}$ using programs made available by David Clayton (http://www-gene.cimr.cam.ac.uk/clayton/ software/stata/ibdreg). As some families had more than two affected sibling pairs (ASPs), the logistic regression analysis was clustered on family and robust variance estimation was used to adjust for familial correlation.

The LOD score associated with this method ((2)) is not directly comparable to a classic LOD score because of the larger number of degrees of freedom. In order to assess the genome-wide significance level, 1000 data sets were therefore simulated under the null hypothesis of no linkage with the same family structures as in the real data using the program MERLIN. ${ }^{17}$ In these simulations, genotypes were randomly assigned to family founders at all markers based on allelic frequencies in the sample, assuming HardyWeinberg and linkage equilibrium. Alleles were then transmitted from one generation to the next according to Mendelian laws using the true genetic map. As we are interested in testing the significance of overall linkage including the covariate effect, only genotypes were randomised whereas the covariate was kept unchanged. The simulated data were analysed using the same method as the real data. In addition to the covariate analysis, the 
original multipoint non-parametric linkage analysis based on $S_{\text {ALL }}$ statistic using the program Allegro ${ }^{15,18}$ was repeated in two subsets of the data: both sibs in the pair with hypercholesterolemia and neither sib in the pair with hypercholesterolemia. The association between hypercholesterolemia, CAD subtypes and other risk factors was examined using $\chi^{2}$ tests for binary factors and a two-sided $t$-test for continuous traits.

\section{Results}

\section{Hypercholesterolemia data}

The clinical data are summarised in Table 1 . The majority of subjects $(3000,72 \%)$ reported themselves as being hypercholesterolemic and taking cholesterol-lowering treatment. Among those who said they were not receiving treatment a further 384 reported themselves to be aware that they had hypercholesterolemia, and 674 indicated that they were not hypercholesterolemic. There were also 44 persons reporting treatment who said they had not been told they were hypercholesterolemic. The remaining 73 did not know their hypercholesterolemic status. As the proportion of somewhat ambiguous self-reports is not high (10.3\%, i.e. 384 who were hypercholesterolemic but not treated and 44 who were non-hypercholesterolemic but under treatment), the analysis was carried out using the entire sample except only the 73 with missing data $(1.7 \%)$. The analysis was thus based on 4102 persons (full sample, i.e. 3384 hypercholesterolemic and 718 non-hypercholesterolemic) (Table 1). This gave rise to 108 pairs of nonhypercholesterolemic sibs, 605 pairs where only one sib was hypercholesterolemic and 1678 pairs where both were hypercholesterolemic. A sensitivity analysis was carried out excluding the $10.3 \%$ with ambiguous self-reports (reduced sample).

\section{Covariate analysis of sibling pairs affected by CAD}

The maximum LOD score obtained in the analysis of ASPs using the full sample and including hypercholesterolemia as a covariate is 4.4 (Figure 1) between markers D2S112 and D2S1326, in the same region as previously. ${ }^{1}$ In 1000

Table 1 Self-reported status on hypercholesterolemia covariate

\begin{tabular}{lrcr}
\hline Hypercholesterolemia $^{\text {a }}$ & Treatment & No treatment & Total \\
\hline Yes & 3000 & 384 & 3384 \\
No & 44 & 674 & 718 \\
Unknown & 5 & 68 & 73 \\
Total & 3049 & 1126 & 4175 \\
\hline
\end{tabular}

aThe 'full sample' consists of the 3384 persons self-reported as hypercholesterolemic and the 718 self-reported as non-hypercholesterolemic. The 'reduced sample' used in sensitivity analysis consists of the $\mathbf{3 0 0 0}$ persons hypercholesterolemic and taking lipid-lowering treatment plus the 674 non-hypercholesterolemic and non-treated. simulations under the null hypothesis of no linkage, at least one LOD score peak $\geqslant 4.4$ was reached 40 times, giving a genome-wide significance of 0.04. (In fact, in each of these 40 simulations only one such peak was observed if, following Holmans et al., ${ }^{12}$ two peaks are considered to define two different linkage regions when they were separated by $30 \mathrm{cM}$ or more.)

At the position of the highest LOD score, the effect of the covariate itself is highly significant. Taking ASPs both of whom are hypercholesterolemic as the baseline, the estimate of the coefficient $\beta_{0}$ for ASPs neither of whom is hypercholesterolemic is 0.66 (95\% confidence interval (CI): 0.37-0.94, $P<0.001$ ), whereas no difference was found in ASPs with one hypercholesterolemic and one non-hypercholesterolemic sibling compared with the same baseline ( $\beta_{1}=0.07,95 \% \mathrm{CI}:-0.09-0.22, P=0.39$, Table 2 ). This indicates greater allele-sharing at this locus in ASPs neither of whom is hypercholesterolemic compared with other ASPs.

\section{Allele sharing estimates and subsets analysis}

Siblings affected by CAD without hypercholesterolemia share on average 1.25 alleles IBD (95\% CI: 1.15-1.36, Table 3 ) at the position of the LOD score peak. This IBD sharing is higher than in siblings both of whom have hypercholesterolemia (1.00 alleles, 95\% CI: 0.98-1.04) and in sibling pairs only one of whom is hypercholesterolemic (1.03 alleles, 95\% CI: 0.99-1.09), neither of which differ significantly from expected sharing under the null hypothesis of no linkage. In summary, only sibling pairs without hypercholesterolemia show evidence of linkage to the

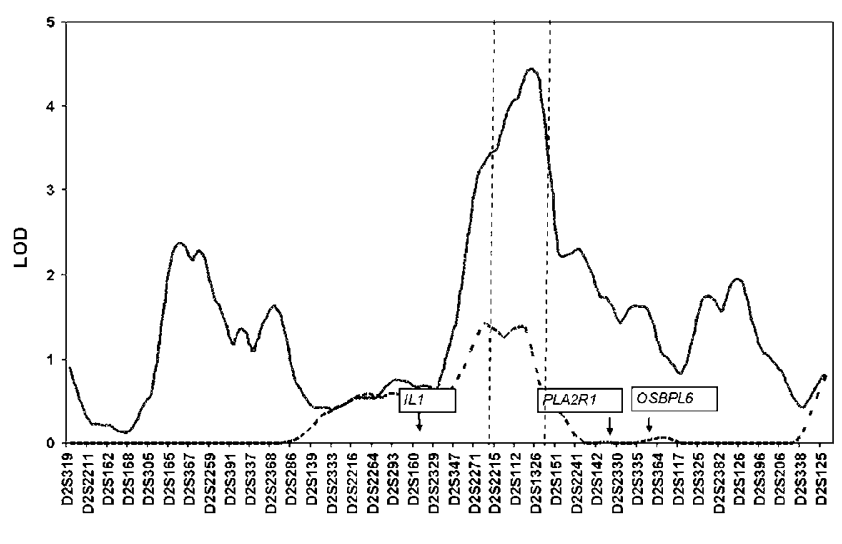

Chromosome 2 markers

Figure 1 LOD scores of linkage to CAD based on the likelihood ratio test including hypercholesterolemia as a covariate (plain line) and not including any covariate (dotted line) on chromosome 2 . The dashed vertical lines indicate the 1-LOD support interval. The position of three candidate genes is indicated. As the markers are not evenly spaced, the $x$ axis is not drawn to scale. Positions of the closest markers to these genes and to the peak LOD score are: $117.9 \mathrm{cM}$ for D2S160, $145.0 \mathrm{cM}$ for $\mathrm{D} 2 \mathrm{~S} 1326,158.8 \mathrm{cM}$ for $\mathrm{D} 2 \mathrm{~S} 142$ and $172.5 \mathrm{cM}$ for D2S335. 
Table 2 Parameter estimates from the linkage analysis with hypercholesterolemia as a covariate at the locus on chromosome 2 showing most evidence of linkage

\begin{tabular}{|c|c|c|c|c|c|c|}
\hline Parameter & $\begin{array}{l}\text { Number of sibs with } \\
\text { hypercholesterolemia }\end{array}$ & $\begin{array}{l}\text { Number of } \\
\text { sibling pairs }\end{array}$ & Effect size & P-value & \multicolumn{2}{|c|}{$95 \% \mathrm{Cl}$} \\
\hline & 2 & 1678 & Baseline & - & - & - \\
\hline$\beta_{0}$ & 0 & 108 & 0.656 & $<0.001$ & 0.369 & 0.944 \\
\hline$\alpha$ & - & & 0.024 & 0.530 & -0.051 & 0.099 \\
\hline
\end{tabular}

Abbreviation: $\mathrm{Cl}$, confidence interval.

Table 3 IBD probabilities and average number of alleles shared at the position of the highest LOD score in sibling pairs affected by $C A D$ according to the number of hypercholesterolemics in the pair

\begin{tabular}{|c|c|c|c|c|c|c|c|c|c|}
\hline & \multicolumn{3}{|c|}{$0^{\mathrm{a}}$} & \multicolumn{3}{|c|}{$1^{\mathrm{a}}$} & \multicolumn{3}{|c|}{$2^{\mathrm{a}}$} \\
\hline & \multirow{2}{*}{$\begin{array}{c}\text { Estimate } \\
0.54\end{array}$} & \multicolumn{2}{|c|}{$95 \% \mathrm{Cl}$} & \multirow{2}{*}{$\begin{array}{c}\text { Estimate } \\
0.51\end{array}$} & \multicolumn{2}{|c|}{$95 \% \mathrm{Cl}$} & \multirow{2}{*}{$\begin{array}{c}\text { Estimate } \\
0.50\end{array}$} & \multicolumn{2}{|c|}{$95 \% \mathrm{Cl}$} \\
\hline$Z_{1}$ & & 0.46 & 0.61 & & 0.48 & 0.54 & & 0.48 & 0.52 \\
\hline$Z_{2}$ & 0.36 & 0.28 & 0.44 & 0.26 & 0.23 & 0.29 & 0.26 & 0.24 & 0.27 \\
\hline$N$-ibd & 1.25 & 1.15 & 1.36 & 1.03 & 0.99 & 1.09 & 1.00 & 0.98 & 1.04 \\
\hline
\end{tabular}

Abbreviations: $\mathrm{Cl}$, confidence interval.

a Headings 0,1 and 2 correspond to the number of hypercholesterolemics in a pair of sibs, $Z_{1}$ and $Z_{2}$ are probabilities of sharing one and two alleles IBD between two sibs, $\mathrm{N}$-ibd=average number of alleles shared IBD between two sibs.

locus on chromosome 2. This is further illustrated by the subset analysis: based on $\mathrm{S}_{\mathrm{ALL}}$ statistic computed using the program Allegro, ${ }^{15,18}$ a maximum LOD score of 3.74 (pointwise $P=0.000017$ ) was obtained at the same locus as previously when only non-hypercholesterolemic pairs were considered, whereas the peak was much smaller in the subset of pairs both of whom are hypercholesterolemic $(\mathrm{LOD}=1.09$, Figure 2 ). In non-hypercholesterolemic patients, the probability of sharing one allele IBD at the locus of the highest LOD is 0.54 (95\% CI: $0.46-0.61)$ and that of sharing 2 alleles IBD is 0.36 (95\% CI: $0.28-0.44$, see Table 3 ). Based on the probability of sharing no allele between affected siblings, ${ }^{19}$ the estimate of the sibling recurrence risk ratio $\left(\lambda_{\mathrm{s}}\right)$ from this locus in non-hypercholesterolemic subjects is 2.5 , although it should be borne in mind that such estimates are approximate and dependent on ascertainment. $^{20,21}$

\section{Sensitivity analysis}

The analysis based on exclusion of subjects with ambiguous reports of hypercholesterolemia status (Table 1) confirmed linkage to the same locus with a LOD score of 4.6 for linkage including hypercholesterolemia as a covariate. The number of ASPs with two non-hypercholesterolemics reduced only slightly, from 108 to 102 (474 ASPs with one hypercholesterolemic and 1340 ASPs with two hypercholesterolemics).

\section{Association between hypercholesterolemia, CAD and its other risk factors}

Table 4 shows that non-hypercholesterolemic subjects are more likely to have had an MI than those with hyper-

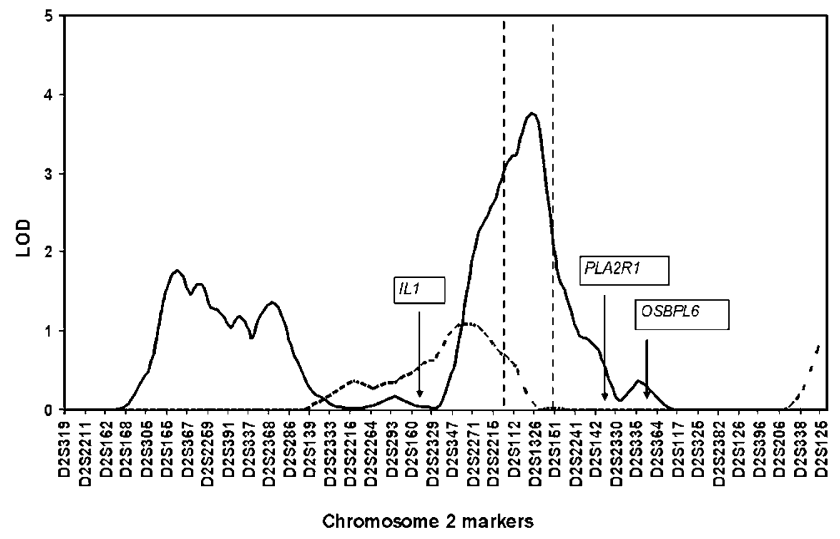

Figure 2 Non-parametric LOD scores computed along chromosome 2 using Allegro ${ }^{15}$ in separate subsets of non-hypercholesterolemic (plain line) and hypercholesterolemic sibling pairs (dotted line). The dashed vertical lines indicate the 1-LOD support interval for the non-hypercholesterolemic pairs subset. The position of three candidate genes is indicated. As the markers are not evenly spaced, the $x$ axis is not drawn to scale. Positions of the closest markers to these genes and to the peak LOD score are: $117.9 \mathrm{cM}$ for D2S160, $145.0 \mathrm{cM}$ for D2S1326, $158.8 \mathrm{cM}$ for D2S142 and $172.5 \mathrm{cM}$ for D2S335.

cholesterolemia (65\% compared with $61 \%, P=0.037$ ). The frequency is higher still in the 108 sibling pairs both of whom are non-hypercholesterolemic (69\%, $P=0.023$, when compared to all hypercholesterolemics). Nonhypercholesterolemics are also more likely to be male, less likely to have diabetes or hypertension, and have a slightly lower BMI and older age at disease onset than hypercholesterolemics, although the differences are small 
Table 4 Association between hypercholesterolemia MI and other risk factors

\begin{tabular}{|c|c|c|c|c|c|}
\hline \multirow[b]{2}{*}{ Phenotype } & \multirow[b]{2}{*}{ Hypercholesterolemia $N=3384$} & \multicolumn{4}{|c|}{ No hypercholesterolemia } \\
\hline & & All subjects $N=718$ & P-value & 108 ASPS & P-value ${ }^{b}$ \\
\hline $\mathrm{MI}^{\mathrm{c}}$ & $2040(60.8)$ & $463(65.0)$ & 0.037 & 145 (68.7) & 0.023 \\
\hline Male sex ${ }^{c}$ & $2437(72.7)$ & $564(78.6)$ & 0.001 & 169 (79.6) & 0.027 \\
\hline Smoking ${ }^{c}$ & 2493 (73.7) & $508(70.9)$ & 0.12 & $144(67.6)$ & 0.051 \\
\hline Diabetes $^{c}$ & $429(12.7)$ & $70(9.7)$ & 0.027 & $24(11.3)$ & 0.536 \\
\hline Hypertension $^{c}$ & $1599(47.9)$ & $283(39.8)$ & $<0.001$ & $93(44.1)$ & 0.282 \\
\hline Age onset ${ }^{d}$ & $52.1(7.6)$ & $53.4(7.3)$ & 0.0001 & $54.0(6.9)$ & 0.0005 \\
\hline$B M l^{d}$ & $27.9(4.2)$ & $27.6(5.9)$ & 0.063 & $27.2(4.3)$ & 0.029 \\
\hline
\end{tabular}

Abbreviation: BMI, body mass index. Owing to small amounts of missing data on some risk factors, the proportions in the cells of this table are based on slightly different totals.

${ }^{a}$ Test of the difference between all hypercholesterolemic and all non-hypercholesterolemic subjects of the sample.

${ }^{b}$ Test of the difference between all hypercholesterolemics and the 108 ASPs both of whom are non-hypercholesterolemic.

${ }^{\mathrm{N}}$ Number (percentage).

${ }^{\mathrm{d}}$ Mean (s.d.).

(Table 4). The association of hypercholesterolemia with other factors raises the possibility that the effect on linkage is attributable to an associated factor or factors, rather than hypercholesterolemia itself. To investigate this each of the factors from Table 4 was considered as an independent covariate in the linkage analysis, but none showed any evidence of heterogeneity associated with the chromosome 2 locus (data not shown). This therefore argues strongly against this explanation.

\section{Discussion}

Genetic heterogeneity has a major impact on the power to map genes for complex diseases. The results reported in this study suggest that the absence of significant evidence of linkage of CAD to chromosome 2 in the original analysis, ${ }^{1}$ despite the large sample size, may be due to genetic heterogeneity associated with hypercholesterolemia. Using hypercholesterolemia as a covariate, we found a locus with a LOD score of 4.4 (Figure 1) with a genome wide $P$-value of 0.04 . The effect size of the covariate is positive and highly significant $(0.66, P<0.001$, see Table 2) in sibling pairs, both of whom are unaffected by hypercholesterolemia compared with sibling pairs both of whom are hypercholesterolemic, and this result was also illustrated by subset analysis and estimation of allele sharing.

Covariate definition is an important issue. In our study, hypercholesterolemia status was based for the majority of subjects on self-report of whether they had ever received treatment for this condition. Treatment may be given as a preventative measure, but we would expect the majority of subjects who have never received treatment to be, or to have been at the time of their diagnosis, free from hypercholesterolemia, although some misclassification is of course possible. Measures of total cholesterol were also available for a small number of subjects, but these measures were taken in most cases while on treatment and also many years (up to 39 years) after their first CAD event, so that they are unlikely to reflect the lipid profile that prevailed when they were first diagnosed and started treatment. We therefore did not make use of these measures in this study.

Six additional covariates (sex, age of onset, diabetes, smoking, hypertension and BMI) were also analysed as potential sources of heterogeneity, but none of these showed any evidence of heterogeneity associated with the chromosome 2 locus (data not shown). As we did not correct for the number of covariates tested, the results presented here should be treated with some caution. However, it is of interest that there is other evidence of linkage of CAD to this region: a LOD score of 3.7 was reported in an isolated Finnish population ${ }^{22}$ and a LOD score of 3.82 was found in the same region using a US cohort of MI patients. ${ }^{7}$ In the Finnish study, ${ }^{22}$ families were excluded when a proband was affected by familial hypercholesterolemia, whereas the US study ${ }^{7}$ excluded all the subjects affected by hypercholesterolemia. Farrall et al. $^{23}$ also found a LOD score $>1$ in the same region using a cohort from across four European countries. In addition to confirming these results reported previously, our study clearly identifies the phenotypic subgroup that is linked to this locus and gives an approximate estimation of the associated $\lambda_{\mathrm{s}}$ in this subgroup.

The commonest recognised mechanism that gives rise to CAD is the accumulation of low-density lipoproteincholesterol (LDL-C) in the arterial wall forming a 'plaque' followed by its rupture and blood clotting leading to areas of increasing arterial obstruction and eventually vessel occlusion. ${ }^{3}$ This process is associated with a number of risk factors, particularly a high level of LDL-C circulating in the blood. In contrast high-density lipoprotein-cholesterol (HDL-C) appears to play a protective role by inhibiting the oxidation of LDL-C and by transporting LDL-C from tissues to the liver where it is metabolised. ${ }^{3}$ Low level of HDL-C is an independent risk factor for CAD. ${ }^{3,24,25}$ 
The results of our study suggest a locus on chromosome 2 that predisposes to CAD in subjects whose cholesterol is not considered to be raised. A pathway independent of cholesterol may then have been exposed in this group. There are many contenders, not least any cause of arterial membrane inflammation (such as infection, blood pressure, hormones, increased level of inflammatory mediators) that can stimulate blood coagulation and artery occlusion. ${ }^{3,26-29}$ A strong candidate locus in the region of interest is the interleukin 1 gene cluster (IL1). An increased level of IL1 cytokines has been repeatedly associated with inflammatory mechanisms, vascular thrombogenicity and arthrosclerosis both in vivo and in vitro. $3,27,29,30$

An alternative is that a group with low HDL-C has been identified. Supporting this possibility are the results of two other studies: using the Framingham Heart Study data set, a quantitative trait locus in the same region was found to be significantly involved in variations of HDL-C levels. ${ }^{31,32}$ Candidate genes that are possibly involved in the regulation of HDL-C levels in this region are PLA2R1 and OSBPL6. ${ }^{31}$ The product encoded by PLA2R 1 is a receptor of the enzyme sPLA2 (secretory phospholipase A2), a major catabolyser of HDL-C in acute and chronic inflammatory conditions. ${ }^{33,34}$ Association has been reported between increased levels of sPLA2 and CAD, particularly angina. ${ }^{35-37}$ The gene OSBPL6 codes for the oxysterol binding proteinlike- 6 receptor. Oxysterols, which bind to this receptor, are products of cholesterol oxidation and their levels are also associated with CAD. ${ }^{38}$ All of these candidate genes lie outside the 1-LOD support interval for the peak LOD score (see figures), but it has been found that linkage peaks often lie quite distant to disease loci. ${ }^{39}$

In summary, we report stronger evidence of linkage to CAD of the initially reported locus on chromosome 2 when allowing for hypercholesterolemia, the evidence for linkage being confined to subjects without hypercholesterolemia. This study illustrates the potential of inclusion of covariates to increase the power of genetic linkage analysis in the presence of heterogeneity. The chromosome 2 locus is a good candidate for further investigation in order to find the actual polymorphisms implicated in the predisposition to CAD in patients unaffected by hypercholesterolemia.

\section{Acknowledgements}

The Family Heart Study was supported by a program grant from the British Heart Foundation (RG2000010). We thank all families who participated in the study, as well as staff in hospitals and general practices throughout the UK who helped in the collection of DNA samples and validation of medical records.

\section{The BHF family heart study research group:}

Leeds

Stephen Ball, Anthony Balmforth, Jenny Barrett, Lynne Barthorpe, Timothy Bishop, Natalie Burtonwood, Richard Cuthbert, Micha
Dorsch, Nigel Durham, Stacey Ellis, Claire Forest, Joanne Fox, Alistair Hall, Vera Hall, Beryl Jackson, Natasha Kelly, Richard Lawrance, Azhar Maqbool, Samantha Mason, Lynne Midgley, Christine Morrell, Jérémie Nsengimana, Julia Oldham, Sarah Pickett, Mark Platts, Natalie Pleasants, Elizabeth Rennie, Adrian Smith, Samantha Thompson, Peter Tooze, Kevin Walters and Nadira Yuldasheva.

Leicester

Sue Adams, Claire Bodycote, Peter Braund, Paul Burton, JennyRebecca Clemitson, Pat de Souza, Rick Dixon, Julie Faulkes, Laura Hopwood, Andrea Koekemoer, Elaine Logtens, Massimo Mangino, Jenny Ogleby, Stuart Raleigh, Cathy Ridge, Nilesh Samani, Katrina Scurrah, Nuala Sheehan, Ravi Singh and Julian Stribling.

\section{References}

1 The BHF Family Heart Study Research Group: A genomewide linkage study of 1933 families affected by premature coronary artery disease: The British Heart Foundation (BHF) Family Heart Study. Am J Hum Genet 2005; 77: 1011-1020.

2 Smith JD, Lusis JA: The allelic structure of common disease. Hum Mol Genet 2002; 11: 2455-2461.

3 Lusis JA, Mar R, Pajukanta P: Genetics of atherosclerosis. Ann Rev Genomics Hum Genet 2004; 5: 189-218.

4 Altmüller J, Palmer JL, Fischer G, Scherb H, Wjst M: Genomewide scans of complex human diseases: true linkage is hard to find. Am J Hum Genet 2001; 69: 936-950.

5 Philippe A, Martinez M, Guilloud-Bataille M et al: Genome-wide scan for autism susceptibility genes. Paris Autism Research International Sibpair Study. Hum Mol Genet 1999; 8: 805-812.

6 International Molecular Genetic Study of Autism Consortium: A full genome screen for autism with evidence for linkage to a region on chromosome 7q. Hum Mol Genet 1998; 7: 571-578.

7 Wang Q, Rao S, Shen GA et al: Premature myocardial infarction: novel susceptibility locus on chromosome 1P34-36 identified by genomewide linkage analysis. Am J Hum Genet 2004; 74: $262-271$.

8 Goddard KAB, Whitte JS, Suarez BK, Catalona WJ, Olson JM: Model-free linkage analysis with covariates confirms linkage of prostate cancer to chromosomes 1 and 4. Am J Hum Genet 2001; 68: $1197-1206$

9 Schaid DJ, McDonnell SK, Thibodeau SN: Regression models for linkage heterogeneity applied to familial prostate cancer. Am $J$ Hum Genet 2001; 68: 1189-1196.

10 Olson JM, Goddard KB, Durek DM: The amyloid precursor proteic locus and very-late-onset Alzheimer disease. Am J Hum Genet 2001; 69: 895-899.

11 Holmans P, Hamshere M, Hollingworth P et al: Genome screen for loci influencing age at onset and rate of decline in late-onset Alzheimer's disease. Am J Med Genet B Neuropsychiatr Genet 2005; 135: 24-32.

12 Holmans P, Zubenko GS, Crowe RR et al: Genomewide significant linkage to recurrent, early-onset major depressive disorder on chromosome 15q. Am J Hum Genet 2004; 74: 1154-1167.

13 Rice J: The role of meta-analysis in linkage studies of complex traits. Am J Med Genet (Neuropsychiatric genetics) 1997; 74: 112-114.

14 Holmans P: Detecting gene-gene interactions using affected sib pair analysis with covariates. Hum Hered 2002; 53: 92-102.

15 Gudbjartsson DF, Jonasson K, Frigge ML, Kong A: Allegro, a new computer program for multipoint linkage analysis. Nat Genet 2000; 25: 12-13.

16 StataCorp: Stata statistical software: Release 9. College station, TX: StataCorp LP, 2005

17 Abecasis RG, Cherny SS, Cookson OW, Cardon RL: Merlin - Rapid analysis of dense genetic maps using sparse gene flow trees. Nat Genet 2002; 30: 97-101.

18 Kong A, Cox NJ: Allele-sharing models: LOD scores and accurate linkage tests. Am J Hum Genet 1997; 61: 1179-1188. 
19 Risch N: Linkage strategies for genetically complex traits. II. The power of affected relative pairs. Am J Hum Genet 1990; 46: 229-241.

20 Olson JM, Cordell JH: Ascertainment bias in the estimation of sibling genetic risk parameters. Genet Epidemiol 2000; 18: 217-235.

21 Cordell JH, Olson JM: Correcting for ascertainment bias of relative-risk estimates obtained using affected-sib-pair linkage data. Genet Epidemiol 2000; 18: 307-321.

22 Pajukanta P, Cargill M, Viitanen L et al: Two loci on chromosomes 2 and $\mathrm{X}$ for premature coronary heart disease identified in earlyand late-settlement populations of Finland. Am J Hum Genet 2000; 67: 1481-1493.

23 Farrall M, Green FR, Peden JF et al: Genomewide mapping of susceptibility to coronary artery disease identifies a novel replicated locus on chromosome 17. PLoS Genetics 2006; 2: e72.

24 Tall AR: Plasma high density lipoproteins. Metabolism and relationship to atherogenesis. J Clin Invest 1990; 86: 379-384.

25 Gordon DJ, Probstfield JL, Garrison RJ et al: High-density lipoprotein cholesterol and cardiovascular disease: Four prospective American studies. Circulation 1989; 79: 8-15.

26 Ross R: Atherosclerosis - an inflammatory disease. N Engl J Med 1999; 340: 115-125.

27 Spronk MHH, Van der Voort D, ten Cate H: Blood coagulation and the risk of atherothrombosis: a complex relationship. BMC Genet 2004; 2: 12

28 Esmon TC: The interactions between inflammation and coagulation. Br J Haematol 2005; 131: 417-430.

29 Kornman SK: Interleukin1 genetics, infammatory mechanisms, and nutrigenetic opportunities to modulate diseases of aging. Am J Clin Nutr 2006; 83 (Suppl): 475S-483S.

30 Waehre T, Ynderstad A, Smith C et al: Increased expression of Interleukin-1 in coronary artery disease with downregulatory effects of HMG-CoA reductase inhibitors. Circulation 2004; 109 : 1966-1972.

31 North KE, Martin LJ, Dyer T, Comuzzie AG, Williams JT: HDL cholesterol in females in the Framingham Heart Study is linked to a region of chromosome 2q. BMC Genet 2003; 4 (Suppl 1): S98.

32 Arya R, Lehman D, Hunt JK et al: Evidence for bivariate linkage of obesity and HDL-C levels in the Framingham Heart Study. BMC Genet 2003; 4 (Suppl1): S52.

33 Tietge UJF, Maugeais C, Lund-Katz S, Grass D, deBeer FC, Rader DJ: Human secretory phospholipase A2 mediates decreased plasma levels of HDL cholesterol and ApoA-I in response to inflammation in human ApoA-I transgenic mice. Arterioscler Thromb Vasc Biol 2002; 22: 1213-1218.

34 Petrovic N, Crove C, Langton PE, Misso NLA, Thompson PJ: A simple assay for a human serum phospholipase A2 that is associated with high density lipoproteins. J Lipid Res 2001; 42: $1706-1718$.

35 Kugiyama K, Ota Y, Takazoe K et al: Circulating levels of secretory type II phospholipase A2 predict coronary events in patients with coronary artery disease. Circulation 1999; 100: 1280-1284.

36 Kugiyama K, Ota Y, Kawano H et al: Increase in plasma levels of secretory type II phospholipase A2 in patients with coronary spastic angina. Cardivasc Res 2000; 47: 159-165.

37 Kugiyama K, Ota Y, Sugiyama S et al: Prognostic value of plasma levels of secretory type II phospholipase A2 in patients with unstable angina pectoris. Am J Cardiol 2000; 86: 718-722.

38 Rimner A, Makdessi AS, Sweidan $\mathrm{H}$ et al: Relevance and mechanism of oxysterol stereospecifity in coronary artery disease. Free Radic Biol Med 2005; 38: 535-544.

39 Roberts SB, Maclean CJ, Neale MC, Eaves LJ, Kendler KS: Replication of linkage studies of complex traits: an examination of variation in location estimates. Am J Hum Genet 1999; 65: 876-884. 